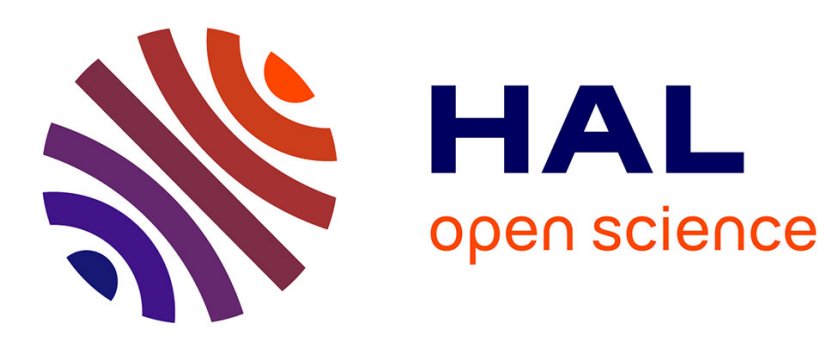

\title{
Histograms of images valued in the manifold of colours endowed with perceptual metrics
}

\author{
Emmanuel Chevallier, Ivar Farup, Jesus Angulo
}

\section{To cite this version:}

Emmanuel Chevallier, Ivar Farup, Jesus Angulo. Histograms of images valued in the manifold of colours endowed with perceptual metrics. Second International Conference on Networked Geometric Science of Information, GSI 2015, Oct 2015, Palaiseau, France. 10.1007/978-3-319-25040-3_81 . hal01121213

\section{HAL Id: hal-01121213 \\ https://hal.science/hal-01121213}

Submitted on 27 Feb 2015

HAL is a multi-disciplinary open access archive for the deposit and dissemination of scientific research documents, whether they are published or not. The documents may come from teaching and research institutions in France or abroad, or from public or private research centers.
L'archive ouverte pluridisciplinaire HAL, est destinée au dépôt et à la diffusion de documents scientifiques de niveau recherche, publiés ou non, émanant des établissements d'enseignement et de recherche français ou étrangers, des laboratoires publics ou privés. 


\title{
Histograms of images valued in the manifold of colours endowed with perceptual metrics
}

\author{
Emmanuel Chevallier ${ }^{1}$, Ivar Farup ${ }^{2}$, and Jesús Angulo ${ }^{1}$ \\ 1 MINES ParisTech, PSL-Research University, \\ CMM-Centre de Morphologie Mathématique, France \\ 2 GjÃ̈̈vik University College, Norway \\ emmanuel.chevallier@mines-paristech.fr
}

\begin{abstract}
We address here the problem of perceptual colour histograms. The Riemannian structure of perceptual distances is measured through standards sets of ellipses, such as Macadam ellipses. We propose an approach based on local Euclidean approximations that enables to take into account the Riemannian structure of perceptual distances, without introducing computational complexity.

Keywords: colour images, image histograms, Riemannian metrics
\end{abstract}

\section{Introduction}

The histogram of the intensities is a fundamental descriptor of a grayscale image. It is one of the most important tools to address problems such as contrast enhancement (by histogram linear stretching or using advanced approaches [1]), image segmentation (by 1D clustering), texture processing [2], image retrieval [3], etc. The standard way of computing a histogram is to cut the value space into regular bins and to count the number of pixels that fall into each bin. However the obtained histogram presents important discontinuities. One thus prefers sometimes to use kernel methods which provide smoother results. One sometimes considers the color space as a part of a three dimensional Euclidean space. Under this assumption, the histogram of a color image can be build in the same way as for gray scale image. However, the distances induced on colours by the human perceptual system cannot be represented by a Euclidean space structure. Observation showed that the perceptual relation between colours is better represented in the framework of Riemannian manifolds. The local metrics of the Riemannian structure are experimentally measured by a set of ellipses, such as the Macadam ellipses [4],BFD-P [5] and RIT-DuPont [6]. This Riemannian structure makes the construction of the histogram difficult. On the one hand, except rare situations, there are no regular tilings of the space. On the other hand, kernel methods have been generalized to Riemannian manifolds in [7], but requires the knowledge of the geodesics. In this paper, we propose an approach that takes into account the Riemannian structure while keeping the computation is the Euclidean framework. Thus we propose a way of building histograms that respects better the perceptual distances than histograms build in Euclidean spaces, without increasing the computation time. 


\section{Image histogram and density estimation}

Let us consider an image $I$ as a map:

$$
I:\left\{\begin{aligned}
D & \rightarrow V \\
p & \mapsto I(p)
\end{aligned}\right.
$$

We have, for instance $V=\mathbb{R}$ for grey-scale images or $V=\mathbb{R}^{n}$ for multispectral images. $D$ is the support space of pixels/voxels, typically a subset of $\mathbb{Z}^{2}$ or $\mathbb{Z}^{3}$. The set of values $\{I(p), p \in D\}$ is interpreted as a set of realizations of a random variable $X$. Let us assume that a reference measure $\mu$ is given on the space $V$. Furthermore, make the strong assumption that the law of $X$ has a density $f$ with respect to $\mu$. The density $f$ is an interesting quantity in image processing.

There are various ways of addressing the problem of probability density estimation. In the Euclidean context the most popular techniques are mainly the histograms, the kernels, and the characteristic function density estimator. The characteristic function density estimator consists in the estimation of the Fourier transform or series of the density. Each of these three techniques can be transported in most of Riemannian manifolds. However, the kernel methods become often significantly simpler than the two others. On the one hand, the histogram requires a regular tiling of the space which is a difficult problem for most of Riemannian manifolds. On the other hand the characteristic function method requires explicit expressions of the eigenfunctions of the Laplacian operator, these functions being known only in a few spaces. For its part, the kernel method only requires the knowledge of geodesic distances. In what follows, we chose to focus on the kernel method. Recall that the kernel method in the Euclidean case has the following form:

$$
f(x)=\frac{1}{k} \sum_{p_{i} \in\{\text { pixels }\}} \frac{1}{\lambda^{n}} K\left(\frac{\left\|x-I\left(p_{i}\right)\right\|}{\lambda}\right)
$$

where $\lambda$ is a scaling parameter, $n$ the dimension of the space, $k$ the number of pixels, and $K: \mathbb{R}_{+} \rightarrow \mathbb{R}_{+}$a map which verifies the following properties: $\int_{\mathbb{R}^{n}} K(\|x\|) d x=1, \int_{\mathbb{R}^{n}} x K(\|x\|) d x=0, \sup (K(x))=K(0)$. In this paper, we assume a supplementary condition of bounded support $K(x>1)=0$.

\section{Basics on Riemannian manifolds}

Let $\mathcal{M}$ be topological space, homeomorphic to an open subset of $\mathbb{R}^{n}$. An homeomorphism is bijective continuous map whose inverse is also continuous. Let $\phi$ be an homeomorphism from $U_{\phi} \subset \mathbb{R}^{n}$ to $\mathcal{M}$. $\phi$ is a parametrization of $\mathcal{M}$. A Riemannian metric is a smooth field of scalar product on $U_{\phi}$. In other words, a Riemannian metric associate a positive definite matrix to each points of $U_{\phi}$. A smooth path is a map $\gamma:[a, b] \rightarrow \mathcal{M}$ such that $\phi^{-1} \circ \gamma$ is piece-wise $C^{1}$. Let $\gamma$ 
be such a path. The Riemannian metric induces a notion of length on smooth path:

$$
L(\gamma)=\int_{a}^{b} \sqrt{\left\langle\left(\phi^{-1} \circ \gamma\right)^{\prime}(t),\left(\phi^{-1} \circ \gamma\right)^{\prime}(t)\right\rangle_{\left(\phi^{-1} \circ \gamma\right)(t)}} d t
$$

Where $\langle., .\rangle_{\left(\phi^{-1} \circ \gamma\right)(t)}$ is the scalar product attached to the point $\left(\phi^{-1} \circ \gamma\right)(t)$. The notion of shortest path between two points induces a distance on $\mathcal{M}$. A shortest path is called a geodesic path and can be seen as straight segments.

The scalar product is entirely determined by its unit ball. Expressed in vector coordinates, the associated unit ball takes the form of an ellipse in two dimensions or of an ellipsoid in three dimensions. Thus, the Riemannian metric is given by a field of ellipses or ellipsoids.

\section{Perceptual metric on colours}

Already Riemann used colour as an illustration of the applicability of his geometry [8], and concrete examples of such colour geometries were developed by Helmholz [9], Schrödinger [10] and Stiles [11].

\subsection{Ellipses, local metric}

The first experimental determination of the field of ellipses describing the Riemannian metric of the colour space was performed by MacAdam [4]. The experiment consisted of about 25000 colour matches with one observer, and the ellipses were derived from the covariance matrices of the repeated observations. Later, it has become common practice to denote ellipses obtained in this manner as JND (just noticeable difference) ellipses or ellipsoids.

Later, another type of experiment has become more commonplace. Pairs of colours that are barely perceptually different, are presented to the observer, who is given the task to estimate the magnitude of the perceptual distance using a set of standard pairs. Ellipses, ellipsoids and metrics obtained in this way are normally denoted supra-threshold ellipses. Examples of supra-threshold color difference based data include BFD-P [5] and RIT-DuPont [6].

\subsection{Global model}

Data sets of measurements provide information on distances through the local metric or through distances between specific colours. A global model provides an analytic expression of the distance between two arbitrary colours. The closest the proposed expression is to the Riemannian perceptual distances, the better the model is. The more conventional procedure for going from a tristimulus space to a space closer linked to a perceptual homogeneous space typically includes the following steps. First, apply a linear transform in the tristimulus space such that the base gets close to the cone fundamentals of the retina. Secondly, perform a non-linear compression of the coordinates (e.g., logarithmic or cubic root) in 
order to mimic the non-linear response of the human visual system. Finally, perform a linear transformation of the resulting coordinates in order to correspond better to the perceptual attributes of colour. Typically, the first coordinate is a weighted sum of the coordinates and represent a lightness correlate, whereas the two other coordinates are weighted differences, and represent colour opponent channels such as, e.g., red-green and blue-yellow.

In order to identify the different parameters of the various transforms, different optimisation criteria are used. In the CIELAB colour space [13], the parameters were optimised such that the lightness should correspond to perceived lightness, and that the Euclidean metric in the resulting space should correspond to perceptual colour differences. For the IPT colour space [14], the parameters were optimised in order to achieve a constant perceived hue along straight radial lines in cylindrical coordinates. It is furthermore reasonably well established that in such perceptual spaces, the Euclidean metric is not the one best corresponding to the perceived colour differences, and other models have been proposed, see, e.g., Luo et al. [15] and Farup [12]. In the hyperbolic models proposed in Farup [12], histograms can be computed using adapted kernels, see [16].

\section{Kernel density estimation using local Euclidean approximations}

In general Riemannian manifolds, computing the distance between two arbitrary points given the metric field is a difficult problem. Indeed, finding the distance is a minimization problem over a set of paths. However, for two close points, the local metric provides a satisfying approximation of the Riemannian distance. A probability density measures the ratio between the probability of an infinitesimal volume element and its volume. It is thus a local notion. The central idea of this section takes advantage of the fact that histograms mainly involves local phenomena. Since in a Riemannian manifold the computation of an histogram does not involve computation of long geodesics, the need of a global model that provides distances between every pairs of colours is of lower importance than in most of applications.

Fig. 1 shows a set of ellipses in the projective $a b$ plane. Let us assume that these ellipses represent the local perceptual metric. Let $c$ be a point where the metric has been measured through the ellipse $\mathcal{E}_{c}$. In a neighborhood of $c$, computing distances using the metric measured at $c$ is a better approximation of the perceptual distance than using the canonical euclidean distance of the $a b$ plane. At a point $p$ where the metric is originally unknown, a metric interpolated from the neighbor points $c_{i}$ has all the odds of being more relevant than the canonical Euclidean metric of the map, see Fiq 1.

Let $d_{R}(p, q)$ be the perceptual distance between color $p$ and color $q . d_{R}(p, q)$ is the Riemannian distance induced by the field of ellipses. Let $\|p-q\|$ be the distance associated with the canonical scalar product of the $a b$ plane, and $\|p-q\|_{c}$ be the distance associated with the scalar product induced by the ellipse $\mathcal{E}_{c}$. Let $B(c, R)$ and $B_{c}(c, R)$ be the respective balls of center $c$ and radius $R$. The 
previous discussion can be formalized as follow. It can be shown that:

$$
\lim _{x \rightarrow c} \frac{\|x-c\|_{c}}{d_{R}(x, c)}=1
$$

while if $\|.\|_{c} \neq\|$.$\| , the equality case being exceptional,$

$$
\lim _{x \rightarrow c} \frac{\|x-c\|}{d_{R}(x, c)} \neq 1
$$

Therefore for such a $c$ there exists $A>0$ such that,

$$
\forall R>0, \exists x \in B(c, R), A<\left|\frac{\| x-c||}{d_{R}(x, c)}-1\right| .
$$

On the other hand there exists a real positive number $R_{c}=R_{c, A}$ such that,

$$
\forall x \in B\left(c, R_{c}\right),\left|\frac{\|x-c\|_{c}}{d_{R}(x, c)}-1\right|<A .
$$

We have

$$
\sup _{B\left(c, R_{c}\right)}\left(\left|\frac{\|x-c\|_{c}}{d_{R}(x, c)}-1\right|\right)<A<\sup _{B\left(c, R_{c}\right)}\left(\left|\frac{\|x-c\|}{d_{R}(x, c)}-1\right|\right) .
$$

Thus for $x \in B\left(c, R_{c}\right),\|x-c\|_{c}$ is preferred to $\|x-c\|$. Consider a kernel $K$ and a scaling parameter $\lambda$ such that

$$
\lambda \leq R_{c} \text { and } B_{c}(c, \lambda) \subset B\left(c, R_{c}\right) .
$$

For $x \in B\left(c, R_{c}\right), K\left(\frac{\|x-c\|_{c}}{\lambda}\right)$ is preferred to $K\left(\frac{\|x-c \mid\|}{\lambda}\right)$. For $x \notin B\left(c, R_{c}\right)$, $K\left(\frac{\|x-c\|_{c}}{\lambda}\right)=K\left(\frac{\|x-c\|}{\lambda}\right)=0$. Thus under these assumptions on the scaling parameter $\lambda$, the histogram

$$
f(x)=\frac{1}{k} \sum_{p_{i} \in\{\text { pixels }\}} \frac{1}{\lambda^{n}} K\left(\frac{\left\|x-I\left(p_{i}\right)\right\|_{I\left(p_{i}\right)}}{\lambda}\right)
$$

is preferred to the classical histogram. We think that the hypothesis on $\lambda$ is reasonable in practice, its validation is a subject of further research. Note that the higher the resolution of the image is, the smaller is $\lambda$ and then the more the hypothesis becomes reasonable.

\subsection{Metric interpolation and Euclidean approximation}

Let $\mathcal{M}$ be topological space, homeomorphic to an open subset of $\mathbb{R}^{n}$. Let $\phi$ be an homeomorphism from $U_{\phi} \subset \mathbb{R}^{n}$ to $\mathcal{M}$. A set of scalar products $G_{c_{i}}$ is given for a set of points $\left(c_{i}\right) \in \mathcal{M}$. We consider here the problem of interpolation of the field of metrics. Let $F_{1}$ and $F_{2}$ be two smooth metric fields that coincide with 
the observed ellipses at the points $\left(c_{i}\right)$. Despite the intuition, if no assumption is made on $\phi$ regarding the Riemannian distance, there are no criteria that enables to prefer $F_{1}$ or $F_{2}$. The problem of metric tensor interpolation is thus a difficult problem. In this paper, we adopt an elementary solution. Ellipses are represented in the projective $a b$ plane. A Delaunay triangulation with respect to the canonical Euclidean metric of the plane is performed on the set $\left(c_{i}\right)$. At a point $p$ in the triangle $c_{i} c_{j} c_{k}$ the parameters of the interpolated ellipse $\mathcal{E}_{p}$ are linearly interpolated between the parameters of $\mathcal{E}_{c_{i}}, \mathcal{E}_{c_{j}}$, and $\mathcal{E}_{c_{k}}$ with respect to the barycentric coordinates of $p$. If $p$ does not belong to one of the triangles of the Delaunay triangulation, we set $\mathcal{E}_{p}=\mathcal{E}_{q}$ where $q$ is the projection of $p$ on the convex hull of the set of centers.

\section{$5.2 \quad$ Experimental results}

The RIT-DuPont dataset [6] shows that the perceptual metric is dependent of the luminance. Nevertheless, for visualization purpose we choose to abandon the luminance information in order to work with two dimensional data. The Macadam ellipses were measured at a fixed luminance, in the CIE chromaticity diagram. The ellipses are transported in the $L=40$ of the $L a b$ space. Forgetting the luminance coordinate, one obtains then a transport of the Macadam ellipses in the projective $a b$ plane. Remind that the proposed framework is independent of the dimension and can be used in three dimensional spaces with standard datasets of ellipsoids.

Fig. 1 presents the transported Macadam ellipses in the projective $a b$ plane, the Delaunay triangulation of the set of centers and the interpolation of the ellipses. Fig. 3 represents the density of the Riemannian measure with respect to the Lebesgue density of the plane. Recall that the expression of the density is given by $\sqrt{\operatorname{det}(G)}$ where $G$ is the metric tensor derived from the ellipse.

Fig. 4 presents the histograms of the image of Fig. 2. (c) and (d) have to aim to study the density $f$ with respect to the perceptual Riemannian volume measure. The main difference between (c) and (d) is that in (d) the shape of the kernel follows the Riemannian metric. The density with respect to the Euclidean measure is visibly different from the histogram with respect the Riemannian measure. The amplitude of the upper spot, representing white colours, is significantly decreased when using the Riemannian measure. Perceptually, this results from the fact that the eyes have an higher sensitivity around white than around blue.

\section{Conclusion}

Given a set of ellipses representing the perceptual metric on colours, we proposed an approach for histogram computation that takes into account the Riemannian structure of the perceptual metric without introducing supplementary computational complexity. Indeed, the step of ellipses interpolation only has to be achieved once and does thus not introduce computational complexity. The relevance of the approach is conditioned by the relevance of the set of perceptual 
ellipses and the quality of the interpolation. The deep problem of metric tensor interpolation has been partially left aside and will be subject of future research. The second topic of our future research will be on the convergence of the proposed histogram to the density of the underlying random variable with respect to the interpolated Riemannian metric.

\section{References}

1. G. Sapiro and V. Caselles. Histogram Modification via Differential Equations. Journal of Differential Equations, 135(2):238-268, 1997.

2. J. Portilla and E.P. Simoncelli. A parametric texture model based on joint statistics of complex wavelet coefficients. International Journal of Computer Vision, 40:47-71, 2000 .

3. Y. Gong and C.H. Chuan and G. Xiaoyi. Image indexing and retrieval using color histograms. Multimedia Tools and Application, 2:133-156, 1996.

4. David L. MacAdam. Visual sensitivities to color differences in daylight. J. Opt. Soc. Am., 32(5):247-274, May 1942.

5. MR Luo and B Rigg. Chromaticity-discrimination ellipses for surface colours. Color Res. Appl., 11(1):25-42, 1986.

6. Roy S Berns, David H Alman, Lisa Reniff, Gregory D Snyder, and Mitchell R Balonon-Rosen. Visual determination of suprathreshold color-difference tolerances using probit analysis. Color Res. Appl., 16(5):297-316, 1991.

7. B. Pelletier. Kernel density estimation on Riemannian manifolds. Statistics and Probability letters, 73(3):297-304, 2005.

8. B Riemann. Ueber die Hypothesen, welche der Geometrie zu Grunde liegen, Abh. Ge. Wiss. Gött, 13(1):133-152, 1868.

9. H von Helmholtz. Versuch einer erweiterten Anwendung des Fechnerschen Gesetzes im farbensystem. Z. Psychol. Physiol. Sinnesorg., 2:1-30, 1891.

10. E. Schrödinger. Grundlinien einer Theorie der Farbenmetrik im Tagessehen (III. Mitteilung). Ann. Phys., 368(22):481-520, 1920.

11. W. S. Stiles. A modified Helmholtz line-element in brightness-colour space. $P$. Phys. Soc., 58:41-65, 1946.

12. Ivar Farup. Hyperbolic geometry for colour metrics. Optics Express, 22(10):1236912378, 2014.

13. Alan R Robertson. The cie 1976 color-difference formulae. Color Research $\&$ Application, 2(1):7-11, 1977.

14. Fritz Ebner and Mark D. Fairchild. Development and testing of a color space (IPT) with improved hue uniformity. In The Sixth Color Imaging Conference: Color Science, Systems and Applications, pages 8-13. IS\&T, 1998.

15. M. Ronnier Luo, Guihua Cui, and B. Rigg. The development of the CIE 2000 colour-difference formula: CIEDE2000. Color Res. Appl., 26(5):340-350, 2001.

16. E. Chevallier, F. Barbaresco, J.Angulo. Probability density estimation on the hyperbolic space applied to radar processing. $H A L,<$ hal-01121090 $>$ 


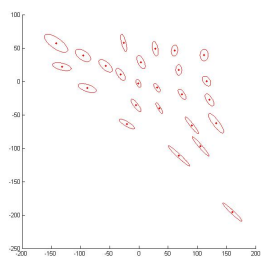

(a)

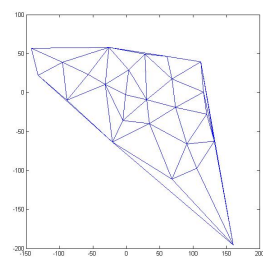

(b)

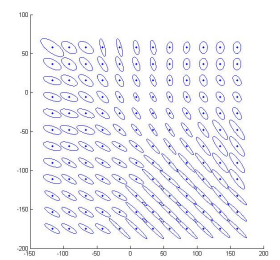

(c)

Fig. 1. (a): Macadam ellipses transported in the projective $a b$ plane, (b): Delaunay triangulation, (c): ellipses interpolation

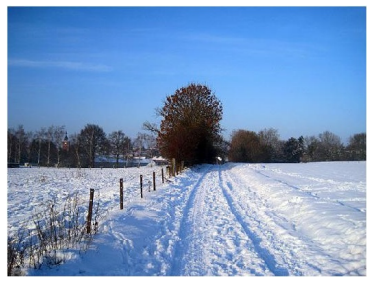

Fig. 2. color photography

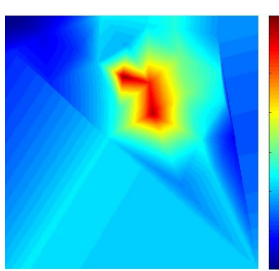

(a)

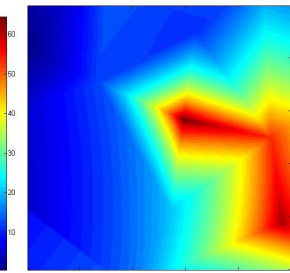

(b)

Fig. 3. (a): local density change induced by the interpolated ellipses, (b): Zoom adapted to colours present in Fig. 2

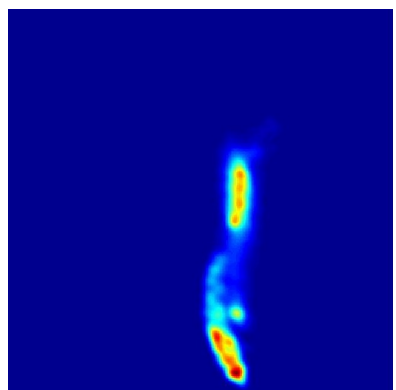

(a)

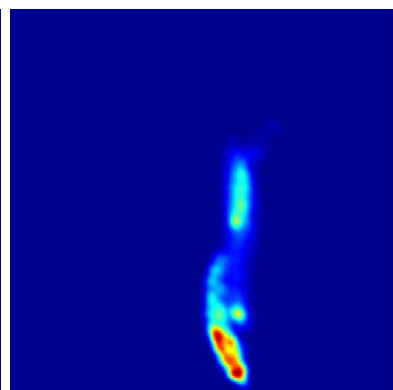

(b)

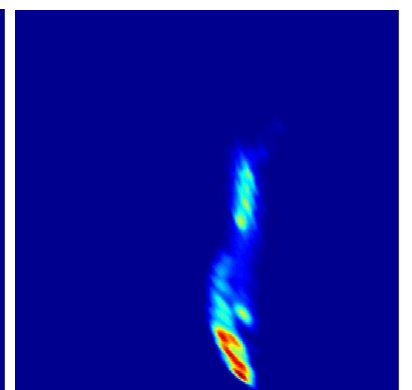

(c)

Fig. 4. The histogram of the image of Fig. 2 is computed using: the canonical Euclidean metric of the $a b$ projective plane in (a), the canonical metric followed by a division by the local density of the perceptual metric in (b) and the formula (3) in (c). 